\title{
Transfer of Catellatospora koreensis Lee et al. 2000 as Catelliglobosispora koreensis gen. nov., comb. nov. and Catellatospora tsunoense Asano et al. 1989 as Hamadaea tsunoensis gen. nov., comb. nov., and emended description of the genus Catellatospora Asano and Kawamoto 1986 emend. Lee and Hah 2002
}

Correspondence

Ismet Ara

ismet@lisci.kitasato-u.ac.jp
Ismet Ara, † Mohammad Abdul Bakir and Takuji Kudo

Japan Collections of Microorganisms, RIKEN BioResource Center, 2-1 Hirosawa, Wako, Saitama 351-0198, Japan

\begin{abstract}
A polyphasic taxonomic analysis of all species of the genus Catellatospora with validly published names revealed morphological, phenotypic and chemotaxonomic heterogeneity. The type species, Catellatospora citrea, and Catellatospora methionotrophica, Catellatospora chokoriensis, Catellatospora coxensis and Catellatospora bangladeshensis had similar morphological and chemotaxonomical properties. Phylogenetic studies based on 16S rRNA gene sequences showed that Catellatospora koreensis and Catellatospora tsunoense were heterogeneous and were also heterogeneous with other species of the genus Catellatospora with respect to the menaquinone composition. DNA-DNA hybridization data showed that the type strains of Catellatospora koreensis and Catellatospora tsunoense were significantly different from each other and other recognized species in the genus Catellatospora. Therefore, on the basis of phenotypic, chemotaxonomic and genomic differences, two new genera with the names Catelliglobosispora gen. nov. and Hamadaea gen. nov. are proposed to accommodate two species classified originally as belonging to the genus Catellatospora and Catelliglobosispora koreensis gen. nov., comb. nov. and Hamadaea tsunoensis gen. nov., comb. nov. are described. The type species of the genus Catelliglobosispora is Catelliglobosispora koreensis (type strain LM $042^{\top}=$ JCM $10976^{\top}=$ DSM $44566^{\top}=$ IMSNU $50729^{\top}$ ) and the type species of the genus Hamadaea is Hamadaea tsunoensis (type strain $6420-\mathrm{P}^{\top}=\mathrm{JCM} 9105^{\top}=\mathrm{DSM} 44101^{\top}=\mathrm{IMSNU}$ $\left.22005^{\top}\right)$.
\end{abstract}

The genus Catellatospora was described by Asano \& Kawamoto (1986) for actinomycete strains that produce

tPresent address: Laboratory of Biological Functions, Department of Drug Discovery Sciences, Kitasato Institute for Life Sciences, Kitasato University, 5-9-1 Shirokane, Minato-ku, Tokyo 108-8641, Japan.

Abbreviations: DAP, diaminopimelic acid; DPG, diphosphatidylglycerol; $\mathrm{OH}-\mathrm{PE}$, hydroxy-phosphatidylethanolamine; $\mathrm{PE}$, phosphatidylethanolamine; PI, phosphatidylinositol; PIM, phosphatidylinositol mannoside.

The GenBank/EMBL/DDBJ accession numbers for the 16S rRNA gene sequences of Catellatospora koreensis IMSNU 50729 ${ }^{\top}$ and Catellatospora tsunoense IMSNU $22005^{\top}$ are AF171700 and AF152110, respectively.

Tables showing $16 \mathrm{~S}$ rRNA gene sequence similarities and chemotaxonomic characteristics of Catelliglobosispora koreensis gen. nov., comb. nov. and Hamadea tsunoensis gen. nov., comb. nov. and recognized species of the genus Catellatospora are available as supplementary material with the online version of this paper. short chains of non-motile spores borne directly on the substrate mycelium without the formation of aerial mycelium. This genus originally contained two species, Catellatospora citrea and Catellatospora ferruginea. Subsequently, Catellatospora citrea subsp. methionotrophica (Asano \& Kawamoto, 1988), Catellatospora matsumotoense, Catellatospora tsunoense (Asano et al., 1989) and Catellatospora koreensis (Lee et al., 2000) were added. Later, Catellatospora matsumotoense was transferred to the genus Micromonospora as Micromonospora matsumotoense, based on 16S rRNA gene sequence analysis and phenotypic characteristics (Lee et al., 1999), and Catellatospora ferruginea (Asano \& Kawamoto, 1986) was transferred to the genus Asanoa as Asanoa ferruginea (Lee \& Hah, 2002). Recently, Catellatospora citrea subsp. methionotrophica (Asano \& Kawamoto, 1988) was transferred to Catellatospora methionotrophica based on DNA-DNA 
hybridization data (Ara \& Kudo, 2006) and Catellatospora bangladeshensis, Catellatospora coxensis and Catellatospora chokoriensis were described (Ara \& Kudo, 2006). The chemical heterogeneity between members of the genus Catellatospora has also been reflected in comparative analysis of 16S rRNA gene sequences (Koch et al., 1996; Lee et al., 1999; Ara \& Kudo, 2006). The chemical heterogeneity among members of this genus was also increased following the descriptions of Catellatospora tsunoense (Asano et al., 1989) and Catellatospora koreensis (Lee et al., 2000). The emergence of molecular systematics, numerical phenetic classification and chemotaxonomy has led to the assignment of seven species, C. citrea, C. methionotrophica, C. bangladeshensis, C. coxensis, C. chokoriensis, C. koreensis and C. tsunoense to the genus Catellatospora (Asano \& Kawamoto, 1986; Asano et al., 1989; Lee et al., 1999; Ara \& Kudo, 2006). The major aim of this study was to re-evaluate the taxonomic status of Catellatospora koreensis and Catellatospora tsunoense using a polyphasic approach and to emend the description of the genus Catellatospora. In addition, on the basis of the results of a polyphasic approach to bacterial classification, we propose the creation of two new genera, Catelliglobosispora gen. nov. and Hamadaea gen. nov.

The type strains of recognized species of the genus Catellatospora were obtained from the Japan Collection of Microorganisms (JCM) and maintained on yeast extract-malt extract agar (ISP medium 2) at $4{ }^{\circ} \mathrm{C}$ and as suspensions in $20 \%(\mathrm{v} / \mathrm{v})$ glycerol at $-20{ }^{\circ} \mathrm{C}$. Strains were grown on tap-water agar and sucrose-nitrate agar (Waksman no. 1) media at $28{ }^{\circ} \mathrm{C}$ for 21 days and then observed using light microscopy and scanning electron microscopy (model S-2400 Hitachi; Tokyo). The sample for scanning electron microscopy was prepared as described by Itoh et al. (1989) and Ara \& Kudo (2007b, c). The phenotypic properties were examined using several standard methods. For cultural characterization, the isolates were grown for 21 days at $28^{\circ} \mathrm{C}$ on various agar media as described by Waksman (1950, 1961), Shirling \& Gottlieb (1966) and Asano \& Kawamoto (1986). The Color Harmony Manual (Jacobson et al., 1958) was used to determine the names and designations of colony colours. The temperature range and $\mathrm{NaCl}$ tolerance for growth were determined on yeast extract-starch agar. Utilization of carbohydrates as sole carbon sources was tested by using neutralized yeast nitrogen base without amino acids as a basal medium according to the method of Stevenson (1967). Production of melanoid pigments was examined using tyrosine agar (ISP medium 7).

For chemotaxonomic analyses, freeze-dried cells were obtained from cultures grown in yeast extract-starch broth on a rotary shaker at $28{ }^{\circ} \mathrm{C}$. The isomers of diaminopimelic acid (DAP) present in the cell wall peptidoglycan were determined by using TLC as described by Staneck \& Roberts (1974). Reducing sugars from whole-cell hydrolysates were analysed using the HPLC method of Mikami \& Ishida (1983). The $N$-acyl group of muramic acid present in the peptidoglycan was determined using the method of Uchida \& Aida (1984). Cell phospholipids were extracted and identified using the method of Minnikin et al. (1984). Methyl esters of cellular fatty acids were prepared and analysed according to the instructions of the Microbial Identification System (Sherlock Microbial Identification System; Hewlett Packard) (Sasser, 1990). Isoprenoid quinones were extracted using the method of Collins et al. $(1977,1984)$ and were analysed by HPLC with a Cosmosil 5C $\mathrm{C}_{18}$ column $(4.6 \times 150 \mathrm{~mm}$; Nacalai Tesque) (Tamaoka et al., 1983) and mass spectrometry (GCMS QP5050; Shimadzu). The preparation and detection of methyl esters of mycolic acids were carried out as described by Tomiyasu (1982).

Genomic DNA extraction, PCR-mediated amplification of the 16S rRNA gene and sequencing of the PCR products were carried out as described by Nakajima et al. (1999) and Ara et al. (2007a, b). The sequences were multiply aligned with selected sequences (Fig. 1) obtained from GenBank/ EMBL/DDBJ by using the CLUSTAL_X program package (Thompson et al., 1997). The alignment was verified manually and adjusted prior to the construction of a phylogenetic tree. A phylogenetic tree constructed using the neighbour-joining method (Saitou \& Nei, 1987) in the PAUP program package (version 4.0b10) (Swofford, 2001) and CLUSTAL_X program was based on the comparison of 1355 nucleotides present in all the strains as a result of elimination of gaps and ambiguous nucleotides from the sequences between positions 34 and 1491 (Escherichia coli position number). Micromonospora chalcea was used as an outgroup. The confidence values of branches of the phylogenetic tree were determined using bootstrap analyses based on 1000 resamplings (Felsenstein, 1985). The values for sequence similarity among Catellatospora strains were calculated manually after pairwise alignment using the CLUSTAL_X package.

DNA was isolated from biomass using the methods of Tamaoka (1994) and Saito \& Miura (1963) with minor modifications as follows: achromopeptidase crude (Wako Pure Chemicals), $N$-acetylmuramidase SG (Seikagaku Kogyo) and lysozyme were used for lysing cells (Kudo et al., 1998). In case cells were not lysed by these enzymes, the cells were freeze-dried and mechanically ground as described by Raeder \& Broda (1985). The G + C content of the DNA was determined using the HPLC method of Tamaoka \& Komagata (1984). An equimolar mixture of nucleotides for analysis of DNA base composition (Yamasa Shoyu, Japan) was digested with bacterial alkaline phosphatase and used as the quantitative standard. DNA-DNA relatedness was measured fluorometrically using the microplate hybridization method devised by Ezaki et al. (1989). Hybridization was carried out at $55{ }^{\circ} \mathrm{C}$ for $2 \mathrm{~h}$.

Almost-complete 16S rRNA gene sequences of the type strains of recognised species were determined and compared with those of representatives of the genera of the family Micromonosporaceae (Ørskov, 1923; Couch, 1950; 


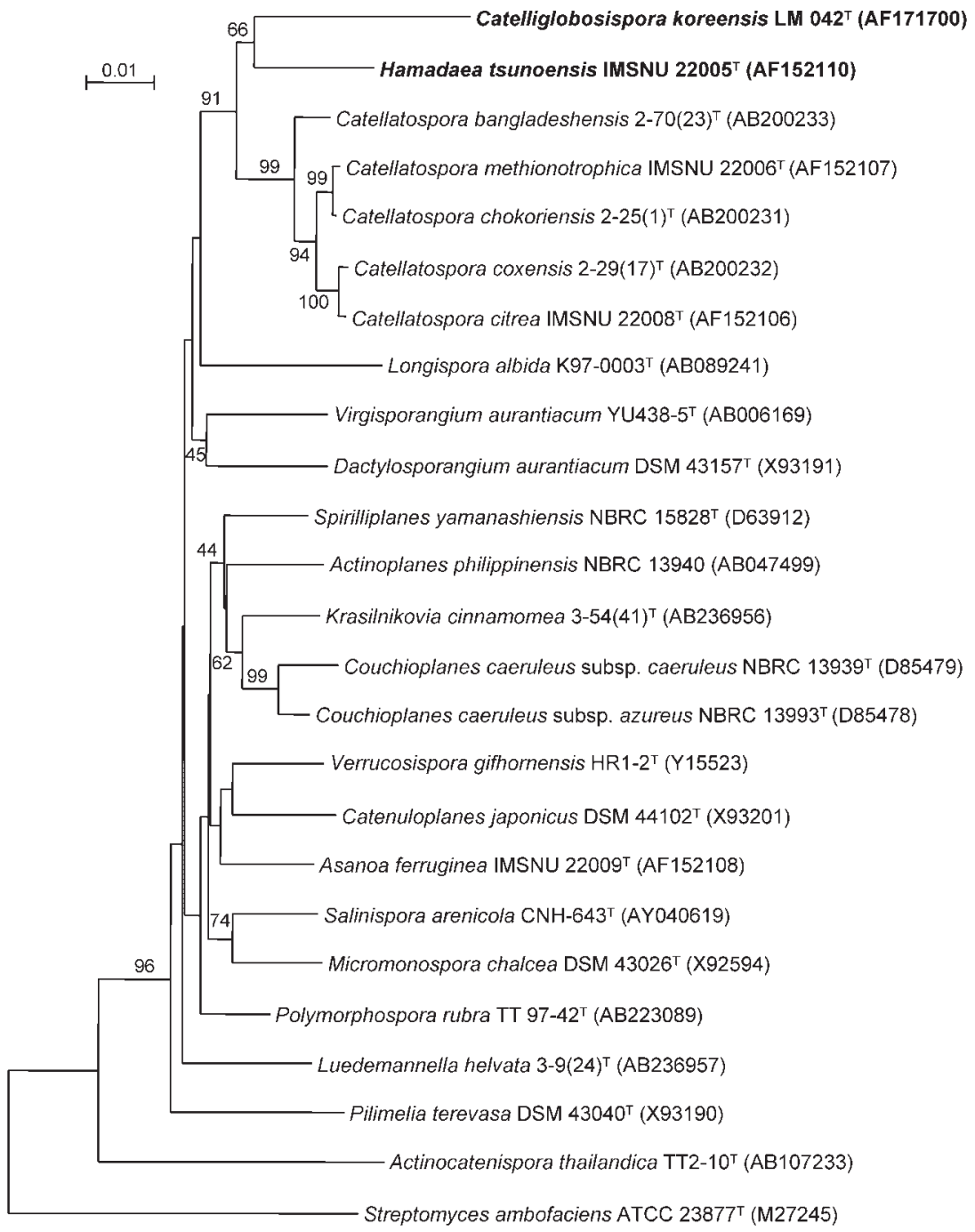

Fig. 1. Phylogenetic tree based on almostcomplete 16S rRNA gene sequences showing the relationships between Hamadaea tsunoensis gen. nov., comb. nov. and Catelliglobosispora koreensis gen. nov., sp. nov. and type species of genera in the family Micromonosporaceae and type strains of the genus Catellatospora. Streptomyces ambofaciens ATCC $23877^{\top}$ was used as an outgroup. The tree was reconstructed by using the neighbourjoining method (Saitou \& Nei, 1987). Bootstrap percentages based on 1000 replications are indicated at branch points (only values $>40 \%$ are given). Bar, 0.01 nucleotide substitutions per 100 nucleotides.
Kane, 1966; Thiemann et al., 1967; Asano \& Kawamoto, 1986; Yokota et al., 1993; Tamura et al., 1994, 1997, 2001, 2006; Rheims et al., 1998; Lee \& Hah, 2002; Matsumoto et al., 2003; Maldonado et al., 2005; Thawai et al., 2006; Ara \& Kudo, 2007a, d). The phylogenetic dendrogram based on the neighbour-joining algorithm (Fig. 1) showed that Catellatospora koreensis and Catellatospora tsunoense formed a distinct clade within the radiation encompassed by the family Micromonosporaceae. The clade was well separated from the $C$. citrea group of the genus Catellatospora and other genera of the family Micromonosporaceae. This relationship was supported by a bootstrap value of $91 \%$ (Fig. 1) and parsimony analysis showed similar topologies for a tree obtained using a distance-based algorithm (data not shown). The two organisms Catellatospora koreensis and Catellatospora tsunoense were distantly associated with the C. citrea clade of the genus Catellatospora, with sequence similarities of 94.7-95.1 and 94.8-96.6\%, respectively (see Supplementary Table S1 available in IJSEM Online).
The chemical properties of Catellatospora koreensis and Catellatospora tsunoense support the conclusion that the two species have been misclassified as members of the genus Catellatospora. Catellatospora koreensis is characterized by the possession of MK-10 $\left(\mathrm{H}_{4}\right)$ (Lee et al., 2000 and this study) and Catellatospora tsunoense by the presence of MK-9 $\left(\mathrm{H}_{6}\right)$ (this study) as major menaquinones (see Supplementary Table S2 in IJSEM Online). Catellatospora tsunoense contained hydroxy-phosphatidylethanolamine (OH-PE) as a diagnostic component of the polar lipids that also included phosphatidylethanolamine (PE), diphosphatidylglycerol (DPG), phosphatidylinositol (PI) and phosphatidylinositol mannosides (PIMs) (Supplementary Table S2 available in IJSEM Online). The phospholipid $\mathrm{OH}-\mathrm{PE}$ was not detected in Catellatospora koreensis or in other members of the genus Catellatospora. On the other hand, members of the $C$. citrea clade in the genus Catellatospora have the major menaquinone $\mathrm{MK}-9\left(\mathrm{H}_{4}\right)$ and are characterized by the presence of meso- and 3-OHDAP and the polar lipids PE, DPG, PI and PIMs 
(Supplementary Table S2). Catellatospora koreensis and Catellatospora tsunoense could be readily differentiated from other members of the genus Catellatospora with regard to the the fatty acid profiles (Table 1). In this study, the fatty acid profiles of members of the genus Catellatospora showed slight differences from the results of Lee et al. (2000). Our results showed that Catellatospora koreensis contained iso- $\mathrm{C}_{16: 0}(44.5 \%)$, iso- $\mathrm{C}_{14: 0}(10.8 \%)$ and iso- $\mathrm{C}_{15: 0}(8.5 \%)$ and that Catellatospora tsunoensis contained iso- $\mathrm{C}_{15: 0}(29.5 \%)$, anteiso- $\mathrm{C}_{17: 0}(15.6 \%)$ and

Table 1. Cellular fatty acid compositions (\%) of Catelliglobosispora koreensis gen. nov., comb. nov., Hamadaea tsunoensis gen. nov., comb. nov. and type strains of the genus Catellatospora

Species: 1, Catelliglobosispora koreensis JCM $10976^{\mathrm{T}}$; 2, Hamadaea tsunoensis JCM $9105^{\mathrm{T}}$; 3, Catellatospora chokoriensis $2-25(1)^{\mathrm{T}} ; 4$, Catellatospora coxensis $2-29(17)^{\mathrm{T}} ; 5$, Catellatospora bangladeshensis 2$70(23)^{\mathrm{T}} ; 6$, Catellatospora citrea JCM $7542^{\mathrm{T}} ; 7$, Catellatospora methionotrophica JCM $7543^{\mathrm{T}}$. Values are percentages of total fatty acids. Values $<1.0 \%$ are not shown.

\begin{tabular}{|c|c|c|c|c|c|c|c|}
\hline Fatty acid & 1 & 2 & 3 & 4 & 5 & 6 & 7 \\
\hline $\mathrm{C}_{15: 0}$ & & 1.0 & 2.2 & 4.8 & 1.7 & 3.9 & 3.0 \\
\hline $\mathrm{C}_{16: 0}$ & 1.2 & 3.1 & & 1.6 & & 1.5 & 1.4 \\
\hline $\mathrm{C}_{17: 0}$ & 1.2 & 7.6 & 10.9 & 14.4 & 3.2 & 8.1 & 8.9 \\
\hline $\mathrm{C}_{18: 0}$ & 2.6 & 2.9 & 4.3 & 1.8 & & 2.2 & 1.7 \\
\hline $\mathrm{C}_{19: 0}$ & & & & 1.2 & & & \\
\hline $2-\mathrm{OH}-\mathrm{C}_{16: 1}$ & 2.9 & & & & & 5.5 & 1.4 \\
\hline $\mathrm{C}_{17: 1} \omega 6 c$ & & 2.7 & & & & & \\
\hline $\mathrm{C}_{17: 1} \omega 8 c$ & & 1.7 & 3.5 & 8.0 & 8.8 & 7.8 & 9.3 \\
\hline $\mathrm{C}_{18: 1} \omega 9 c$ & & & & 1.6 & 3.1 & 2.2 & 3.0 \\
\hline iso- $\mathrm{C}_{14: 0}$ & 10.8 & & 3.7 & 4.8 & 5.2 & 2.4 & 1.5 \\
\hline iso- $\mathrm{C}_{15: 0}$ & 8.5 & 29.5 & 30.3 & 22.2 & 19.8 & 28.4 & 37.6 \\
\hline anteiso- $\mathrm{C}_{15: 0}$ & 3.7 & 8.8 & 6.3 & 7.4 & 2.5 & 5.3 & 7.5 \\
\hline iso- $\mathrm{C}_{15: 1}$ & & & & & & 2.6 & \\
\hline iso- $\mathrm{C}_{16: 1}$ & 8.6 & & 2.4 & & 3.7 & 1.8 & \\
\hline iso- $\mathrm{C}_{16: 0}$ & 44.5 & 10.4 & 22.9 & 18.5 & 35.4 & 10.5 & 5.7 \\
\hline $\begin{array}{l}\text { anteiso- } \\
\mathrm{C}_{17: 1} \omega 9 c\end{array}$ & 1.5 & & & & & & \\
\hline iso- $\mathrm{C}_{17: 1} \omega 9 c$ & & & & & 1.8 & 1.4 & 3.2 \\
\hline iso- $\mathrm{C}_{17: 0}$ & 1.7 & 5.4 & 5.5 & 2.2 & 2.8 & 3.3 & 4.5 \\
\hline anteiso- $\mathrm{C}_{17: 0}$ & 6.6 & 15.6 & 7.9 & 4.5 & 2.1 & 4.3 & 5.0 \\
\hline iso- $\mathrm{C}_{18: 0}$ & 3.0 & & & & 1.2 & & \\
\hline $\begin{array}{l}\text { 10-Methyl } \\
\mathrm{C}_{17: 0}\end{array}$ & & 3.6 & & & 2.3 & 1.0 & \\
\hline $\begin{array}{l}\text { 10-Methyl } \\
\mathrm{C}_{18: 0}\end{array}$ & & 1.5 & & & & & \\
\hline $\begin{array}{l}\text { Summed } \\
\text { feature } 3^{*}\end{array}$ & & 2.0 & & & 1.2 & & \\
\hline $\begin{array}{l}\text { Summed } \\
\text { feature } 6^{*}\end{array}$ & & & & & & 1.5 & 1.4 \\
\hline
\end{tabular}

*Summed features represent groups of one or two fatty acids, which could not be separated by GLC with the MIDI system. Summed feature 3 contains 2-OH-i- $\mathrm{C}_{15: 0}$ and/or $\mathrm{C}_{16: 1} \omega 7 \mathrm{c}$. Summed feature 6 contains $\mathrm{C}_{19: 1} \omega 11 c$ and/or $\mathrm{C}_{19: 1} \omega 9 c$. iso- $\mathrm{C}_{16: 0}(10.4 \%)$ as major fatty acids. Similar fatty acid profiles were not detected in the Catellatospora citrea clade (Table 1). In addition, 10-methyl branched fatty acids were detected in Catellatospora tsunoense but not in the Catellatospora koreensis clade (Table 1). The other members of the genus Catellatospora contained large amounts of iso- $\mathrm{C}_{15: 0}$ and iso- $\mathrm{C}_{16: 0}$; this combination of fatty acids corresponds to fatty acid type 3b (Kroppenstedt, 1985). Catellatospora koreensis and Catellatospora tsunoense could be distinguished from members of other genera of the family Micromonosporaceae by using a combination of chemical characteristics (Table 2) and phylogenetic evidence, supporting the conclusion that these two organisms merit the recognition of two new genera.

Catellatospora koreensis and Catellatospora tsunoense produced a well-developed and branched substrate mycelium. Aerial mycelium was not formed on any agar media tested (Asano et al., 1989; Lee et al., 2000). Straight and relatively short chains of non-motile spores were borne directly on substrate mycelium. Each spore was cylindrical and its surface was smooth. Abundant globose bodies were observed on oatmeal agar for Catellatospora koreensis (Lee et al., 2000), which were morphologically similar to the globose bodies of Catellatospora tsunoense (Asano et al., 1989). It has been reported previously that globose bodies of Catellatospora tsunoense, which were morphologically similar to those of members of the genus Dactylosporangium described by Thiemann et al. (1967) and Sharples \& Williams (1974) and were 0.4-0.6 $\mu \mathrm{m}$ in diameter. Catellatospora tsunoense sporulated only on tapwater agar and calcium-malate agar medium in 10 days to 1 month at $28{ }^{\circ} \mathrm{C}$. It has been reported previously (Asano et al., 1989; Lee \& Hah, 2002) and in the present study that the level of sporulation decreased in cultures grown on poor media compared with cultures grown on rich complex organic media (Table 3). The genus Catellatospora clearly differs from other genera in the actinoplanate group by the morphology of the linear spore units formed directly from the vegetative mycelia. However, globose bodies were not observed in other species of the genus Catellatospora (Asano \& Kawamoto, 1986; Asano et al., 1989; Lee et al., 2000; Ara \& Kudo, 2006).

Physiologically, Catellatospora koreensis and Catellatospora tsunoense could be differentiated from each other by their utilization of D-arabinose, $\alpha$-melibiose, L-rhamnose, trehalose, lactose and raffinose; growth requirement for thiamine; growth on $0.0001 \%$ crystal violet; resistance to $50 \mu \mathrm{g}$ gentamicin $\mathrm{ml}^{-1}$; and growth at $37^{\circ} \mathrm{C}$ and in $1 \% \mathrm{NaCl}$ (Asano et al., 1989; Lee et al., 2000) (Table 4). In the physiological tests described in this study, Catellatospora koreensis and Catellatospora tsunoense were different from other Catellatospora strains tested (Asano et al., 1989; Lee et al., 2000) (Table 4). Catellatospora koreensis and Catellatospora tsunoense were further compared by using DNA-DNA hybridization with other Catellatospora species (Table 5). Catellatospora koreensis and Catellatospora 
Table 2. Morphological features and chemotaxonomic characteristics of Hamadaea gen. nov., Catelliglobosispora gen. nov. and other genera of the family Micromonosporaceae

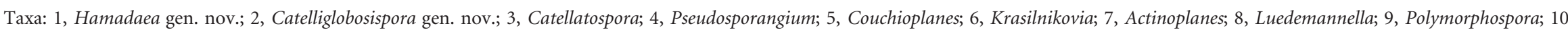

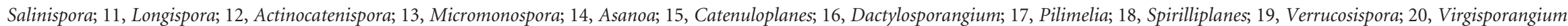

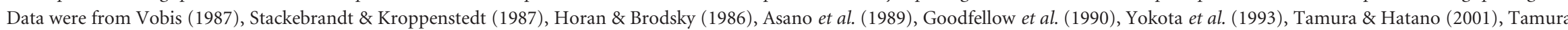

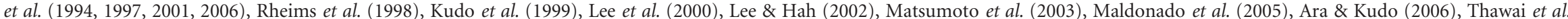
(2006), Ara \& Kudo (2007a) and Ara \& Kudo (2007d). +, Present; -, absent; ND, not determined.

\begin{tabular}{|c|c|c|c|c|c|c|c|c|c|c|c|c|c|c|c|c|c|c|c|c|}
\hline Characteristic & 1 & 2 & 3 & 4 & 5 & 6 & 7 & 8 & 9 & 10 & 11 & 12 & 13 & 14 & 15 & 16 & 17 & 18 & 19 & 20 \\
\hline Sporangium & - & - & - & - & - & - & + & + & - & - & - & - & - & - & - & + & + & - & - & + \\
\hline $\begin{array}{l}\text { Spore } \\
\text { motility }\end{array}$ & - & - & - & - & + & - & + & - & - & - & - & - & - & - & + & + & + & + & - & - \\
\hline $\begin{array}{l}\text { Diamino } \\
\text { acid }\end{array}$ & $\begin{array}{c}\text { meso- and 3- } \\
\text { OH-DAP }\end{array}$ & meso-DAP & meso-DAP & $\begin{array}{l}\text { meso- and } \\
3 \mathrm{OH}-\mathrm{DAP}\end{array}$ & L-lysine & meso-DAP & $\begin{array}{r}\text { meso- } \\
\text { DAP }\end{array}$ & meso-DAP & $\begin{array}{r}\text { meso- } \\
\text { DAP }\end{array}$ & meso-DAP & meso-DAP & $\begin{array}{r}\text { meso- } \\
\text { DAP }\end{array}$ & meso-DAP & meso-DAP & L-lysine & meso-DAP & $\begin{array}{r}\text { meso- } \\
\text { DAP }\end{array}$ & $\begin{array}{c}\text { meso- } \\
\text { DAP }\end{array}$ & $\begin{array}{r}\text { meso- } \\
\text { DAP }\end{array}$ & $\begin{array}{c}\text { meso- } \\
\text { DAP }\end{array}$ \\
\hline $\begin{array}{l}\text { Whole-cell } \\
\text { sugars* }\end{array}$ & $\begin{array}{l}\text { Xyl, Gal, } \\
\text { Man, Rib, } \\
\text { Ara, Rham, } \\
\text { Glu }\end{array}$ & $\begin{array}{l}\text { Rham, Man, } \\
\text { Xyl, Gal, Glu }\end{array}$ & $\begin{array}{c}\text { Xyl, Man, } \\
\text { Gal, Ara, } \\
\text { Rham, } \\
\text { Rib }\end{array}$ & $\begin{array}{c}\text { Gal, Glc, Rib, } \\
\text { Man, Xyl, } \\
\text { Ara }\end{array}$ & $\begin{array}{l}\text { Ara, Gal, } \\
\text { Xyl }\end{array}$ & $\begin{array}{c}\text { Gal, Man, } \\
\text { Xyl, Ara, } \\
\text { Rib }\end{array}$ & Ara, Xyl & $\begin{array}{c}\text { Xyl, Gal, } \\
\text { Man, } \\
\text { Rham, } \\
\text { Rib, Ara }\end{array}$ & Xyl & $\begin{array}{c}\text { Ara, Gal, } \\
\text { Xyl }\end{array}$ & $\begin{array}{l}\text { Ara, Gal, } \\
\text { Xyl }\end{array}$ & $\begin{array}{c}\text { Ara, Gal, } \\
\text { Xyl }\end{array}$ & Ara, Xyl & Ara, Gal, Xyl & Xyl & Ara, Xyl & $\begin{array}{l}\text { Ara, } \\
\text { Xyl }\end{array}$ & Ara, Xyl & $\begin{array}{r}\text { Man, } \\
\text { Xyl }\end{array}$ & $\begin{array}{c}\text { Ara, Gal, } \\
\text { Xyl }\end{array}$ \\
\hline $\begin{array}{l}\text { Fatty-acid } \\
\text { type } \dagger\end{array}$ & $3 \mathrm{~b}$ & $3 \mathrm{~b}$ & $3 \mathrm{~b}$ & $2 \mathrm{~d}$ & $2 \mathrm{c}$ & $2 \mathrm{~d}$ & $2 \mathrm{~d}$ & $2 \mathrm{~d}$ & $2 a$ & $3 a$ & $2 \mathrm{~d}$ & $3 \mathrm{~b}$ & $3 \mathrm{~b}$ & $2 \mathrm{~d}$ & $2 \mathrm{c}$ & $3 \mathrm{~b}$ & $2 \mathrm{~d}$ & $2 \mathrm{~d}$ & $2 \mathrm{~b}$ & $2 \mathrm{~d}$ \\
\hline $\begin{array}{l}\text { Major mena- } \\
\text { quinone(s) } \\
\text { (MK-) }\end{array}$ & $9\left(\mathrm{H}_{6}\right)$ & $10\left(\mathrm{H}_{4}\right)$ & $9\left(\mathrm{H}_{4}\right)$ & $9\left(\mathrm{H}_{6}\right)$ & $9\left(\mathrm{H}_{4}\right)$ & $9\left(\mathrm{H}_{6,4,8}\right)$ & $\begin{array}{l}9\left(\mathrm{H}_{4}\right), \\
10\left(\mathrm{H}_{4}\right)\end{array}$ & $9\left(\mathrm{H}_{6,4,2,8}\right)$ & $\begin{array}{r}10\left(\mathrm{H}_{6,4}\right) \\
9\left(\mathrm{H}_{6,4)}\right)\end{array}$ & $9\left(\mathrm{H}_{4}\right)$ & $10\left(\mathrm{H}_{4,6}\right)$ & $9\left(\mathrm{H}_{4,6}\right)$ & $\begin{array}{r}10\left(\mathrm{H}_{4,6}\right) \\
9\left(\mathrm{H}_{4,6}\right)\end{array}$ & $10\left(\mathrm{H}_{6,8}\right)$ & $\begin{array}{l}9\left(\mathrm{H}_{8}\right), \\
10\left(\mathrm{H}_{8}\right)\end{array}$ & $9\left(\mathrm{H}_{4,6,8}\right)$ & $9\left(\mathrm{H}_{4,2}\right)$ & $10\left(\mathrm{H}_{4}\right)$ & $9\left(\mathrm{H}_{4}\right)$ & $10\left(\mathrm{H}_{4,6,8}\right)$ \\
\hline $\begin{array}{l}\text { Phospholipid } \\
\text { type } ¥\end{array}$ & PII & PII & PII & PII & PII & PII & PII & PII & PII & PII & PII & PII & PII & PII & PIII & PII & PII & PII & PII & PII \\
\hline $\begin{array}{c}\text { DNA G +C } \\
\text { content } \\
(\mathrm{mol} \%)\end{array}$ & 70 & 70 & 70-71 & 73 & 70-72 & 71 & $72-73$ & 71 & 71 & $70-73$ & 70 & 72 & 71-72 & 71-72 & $71-73$ & $71-73$ & ND & 69 & 70 & 71 \\
\hline
\end{tabular}

* Ara, arabinose; Gal, galactose; Man, mannose; Rham, rhamnose; Rib, ribose; Xyl, xylose.

$\dagger$ According to the classification of Kroppenstedt (1985).

¥According to the classification of Lechevalier et al. (1981). 


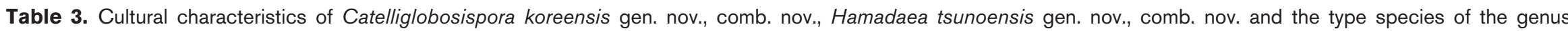
Catellatospora

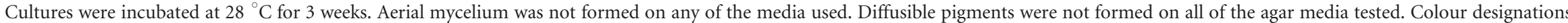

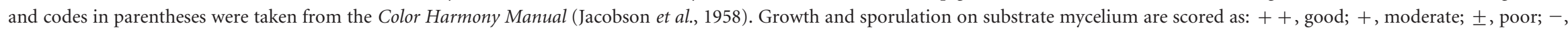
no growth and no spores formed.

\begin{tabular}{|c|c|c|c|c|c|c|c|c|c|}
\hline \multirow[t]{2}{*}{ Agar medium } & \multicolumn{3}{|c|}{ Catelliglobosispora koreensis JCM $10976^{\mathrm{T}}$} & \multicolumn{3}{|c|}{ H. tsunoensis JCM $9105^{\mathrm{T}}$} & \multicolumn{3}{|c|}{ Catellatospora citrea JCM $7542^{\mathrm{T}}$} \\
\hline & Growth & Reverse colour & Sporulation & Growth & Reverse colour & Sporulation & Growth & Reverse colour & Sporulation \\
\hline $\begin{array}{l}\text { Glucose- } \\
\text { asparagine }\end{array}$ & ++ & Colourless & - & \pm & Colourless & - & + & Cream $\left(1 \frac{1}{2} \mathrm{ca}\right)$ & - \\
\hline $\begin{array}{l}\text { Glycerol- } \\
\text { asparagine }\end{array}$ & \pm & Colourless & - & \pm & Colourless & + & + & Cream $\left(1 \frac{1}{2} \mathrm{ca}\right)$ & - \\
\hline Salts-starch & ++ & Honey gold (2ic) & - & \pm & Colourless & - & ++ & $\begin{array}{l}\text { Light wheat } \\
\text { (2ea) }\end{array}$ & - \\
\hline Tyrosine & \pm & Cream $\left(1 \frac{1}{2} \mathrm{ca}\right)$ & + & \pm & $\begin{array}{l}\text { Light wheat } \\
\text { (2ea) }\end{array}$ & - & + & Light $\tan (3 g c)$ & ++ \\
\hline Nutrient & \pm & Cream $\left(1 \frac{1}{2} \mathrm{ca}\right)$ & - & \pm & Colourless & - & \pm & $\begin{array}{l}\text { Light melon } \\
\text { yellow (3ea) }\end{array}$ & - \\
\hline $\begin{array}{l}\text { Yeast-malt } \\
\text { extract }\end{array}$ & + & Light amber (3ic) & - & ++ & Orange (4la) & - & ++ & Orange (4la) & - \\
\hline Oatmeal & ++ & Light amber (3ic) & - & ++ & $\begin{array}{l}\text { Bright maize } \\
\text { (3la) }\end{array}$ & - & ++ & $\begin{array}{l}\text { Light maize } \\
(2 \mathrm{ea})\end{array}$ & + \\
\hline Bennett & ++ & Bamboo $(2 g c)$ & - & ++ & $\begin{array}{l}\text { Bright marigold } \\
(3 \mathrm{pa})\end{array}$ & - & ++ & $\begin{array}{l}\text { Bright marigold } \\
\text { (3pa) }\end{array}$ & - \\
\hline $\begin{array}{l}\text { 1/5 yeast } \\
\text { extract-starch }\end{array}$ & + & $\begin{array}{l}\text { Oatmeal sand } \\
(2 \mathrm{ec})\end{array}$ & - & ++ & Maize (3ga) & + & + & $\begin{array}{l}\text { Light wheat } \\
(2 \mathrm{ea})\end{array}$ & + \\
\hline Oatmeal-nitrate & + & $\begin{array}{l}\text { Oatmeal sand } \\
(2 \mathrm{ec})\end{array}$ & - & + & $\begin{array}{l}\text { Light wheat } \\
\text { (2ea) }\end{array}$ & - & + & Cream $\left(1 \frac{1}{2} \mathrm{ca}\right)$ & + \\
\hline $\begin{array}{l}\text { Glucose-yeast } \\
\text { extract }\end{array}$ & ++ & Honey gold (2ic) & - & ++ & $\begin{array}{l}\text { Bright marigold } \\
(3 \mathrm{pa})\end{array}$ & - & ++ & $\begin{array}{l}\text { Russet orange } \\
(4 \mathrm{pc})\end{array}$ & - \\
\hline Hickey-Tresner & ++ & Light $\tan (3 g c)$ & - & + & Maize (2ga) & - & \pm & $\begin{array}{l}\text { Melon yellow } \\
\text { (3ga) }\end{array}$ & - \\
\hline Tap-water & \pm & Colourless & ++ & \pm & Colourless & ++ & \pm & Colourless & ++ \\
\hline Sucrose-nitrate & \pm & Colourless & ++ & \pm & Colourless & - & \pm & Colourless & ++ \\
\hline $\begin{array}{l}\text { Yeast extract- } \\
\text { starch }\end{array}$ & ++ & Honey gold (2ic) & - & ++ & $\begin{array}{l}\text { Bright marigold } \\
\text { (3pa) }\end{array}$ & - & ++ & $\begin{array}{l}\text { Bright marigold } \\
(3 \mathrm{pa})\end{array}$ & - \\
\hline
\end{tabular}


Table 4. Physiological characteristics of Hamadaea tsunoensis gen. nov., comb. nov., Catelliglobosispora koreensis gen. nov., comb. nov. and type strains of recognized Catellatospora species

Species: 1, H. tsunoensis JCM $9105^{\mathrm{T}} ; 2$, Catelliglobosispora koreensis JCM $10976^{\mathrm{T}} ; 3$, Catellatospora chokoriensis $2-25(1)^{\mathrm{T}} ; 4$, Catellatospora coxensis $2-29(17)^{\mathrm{T}} ; 5$, Catellatospora bangladeshensis $2-70(23)^{\mathrm{T}} ; 6$, Catellatospora citrea JCM $7542^{\mathrm{T}} ; 7$, Catellatospora methionotrophica JCM $7543^{\mathrm{T}}$. Cultures were incubated at $30{ }^{\circ} \mathrm{C}$ for 3 weeks. Growth: ++ , abundant; + , positive; \pm , weakly positive; - , no growth observed.

\begin{tabular}{|c|c|c|c|c|c|c|c|}
\hline Characteristic & 1 & 2 & 3 & 4 & 5 & 6 & 7 \\
\hline \multicolumn{8}{|l|}{ Utilization of: } \\
\hline Glycerol & \pm & - & - & + & \pm & + & \pm \\
\hline Erythritol & - & - & - & - & - & - & \pm \\
\hline Adonitol & - & - & \pm & - & \pm & - & - \\
\hline L-Arabinose & ++ & ++ & ++ & ++ & ++ & ++ & ++ \\
\hline D-Ribose & - & - & - & + & - & + & - \\
\hline D-Xylose & ++ & ++ & ++ & + & ++ & ++ & ++ \\
\hline D-Fructose & ++ & ++ & - & \pm & - & + & - \\
\hline L-Rhamnose & ++ & - & - & ++ & + & + & ++ \\
\hline myo-Inositol & - & - & - & - & - & \pm & \pm \\
\hline D-Mannitol & - & - & - & - & \pm & \pm & - \\
\hline Methyl $\alpha$-D-glucoside & - & - & - & \pm & - & + & - \\
\hline Salicin & \pm & - & ++ & - & ++ & + & ++ \\
\hline D-Lactose & ++ & ++ & ++ & ++ & ++ & ++ & + \\
\hline$\alpha$-(+)-D-Melibiose & ++ & - & ++ & ++ & ++ & + & - \\
\hline Sucrose & ++ & ++ & + & ++ & ++ & ++ & ++ \\
\hline$(+)$-D-Raffinose & ++ & ++ & - & - & - & - & \pm \\
\hline Trehalose & ++ & - & ++ & ++ & ++ & ++ & ++ \\
\hline $\begin{array}{l}\text { Temperature range for } \\
\text { growth }\left({ }^{\circ} \mathrm{C}\right)\end{array}$ & $20-30$ & $15-37$ & $15-30$ & $20-30$ & $25-30$ & $15-30$ & $20-30$ \\
\hline $\mathrm{pH}$ range for growth & $6-9$ & $6-9$ & $6-9$ & $6-9$ & $6.8-7.2$ & $6-9$ & $6.8-7.2$ \\
\hline Growth in $1 \% \mathrm{NaCl}$ & - & + & + & - & - & - & - \\
\hline
\end{tabular}

tsunoense exhibited very low levels of DNA-DNA relatedness to the other Catellatospora species tested (2-7\%), as well as to each other $(2-5 \%)$ (Table 5$)$. Concerning the fatty acid profiles, these organisms showed significant differences in the amounts of iso- $\mathrm{C}_{16: 0}$, iso-C-15:0 and anteiso- $\mathrm{C}_{17: 0}$. In addition, C. koreensis contained iso- $\mathrm{C}_{14: 0}$, iso- $\mathrm{C}_{16: 1}$, iso$\mathrm{C}_{18: 0}, 2-\mathrm{OH}-\mathrm{C}_{16: 1}$ and iso- $\mathrm{C}_{17: 1} \omega 9 c$, whereas 10-methyl$\mathrm{C}_{17: 0}, 10$-methyl- $\mathrm{C}_{18: 0}$, summed feature $3, \mathrm{C}_{17: 1} \omega 6 c$,

Table 5. DNA G $+\mathrm{C}$ content and DNA-DNA relatedness among Catelliglobosispora koreensis gen. nov., comb. nov., Hamadaea tsunoensis gen. nov., comb. nov. and type strains of the genus Catellatospora

\begin{tabular}{|c|c|c|c|c|c|c|c|c|}
\hline \multirow[t]{2}{*}{ Strain } & \multirow{2}{*}{$\begin{array}{c}\mathrm{G}+\mathrm{C} \text { content } \\
(\mathrm{mol} \%)\end{array}$} & \multicolumn{7}{|c|}{ Hybridization (\%) with ${ }^{3} \mathrm{H}$-labelled DNA from: } \\
\hline & & 1 & 2 & 3 & 4 & 5 & 6 & 7 \\
\hline $\begin{array}{l}\text { 1. Catellatospora chokoriensis } \\
2-25(1)^{\mathrm{T}}\end{array}$ & 71 & 100 & 53 & 26 & 66 & 50 & 7 & 4 \\
\hline $\begin{array}{l}\text { 2. Catellatospora coxensis } \\
2-29(17)^{\mathrm{T}}\end{array}$ & 71 & 27 & 100 & 14 & 20 & 31 & 4 & 2 \\
\hline $\begin{array}{l}\text { 3. Catellatospora banglade- } \\
\text { shensis } 2-70(23)^{\mathrm{T}}\end{array}$ & 71 & 26 & 35 & 100 & 30 & 31 & 6 & 5 \\
\hline $\begin{array}{l}\text { 4. Catellatospora citrea JCM } \\
7542^{\mathrm{T}}\end{array}$ & 71 & 50 & 47 & 28 & 100 & 44 & 6 & 5 \\
\hline $\begin{array}{l}\text { 5. Catellatospora methiono- } \\
\text { trophica JCM } 7543^{\mathrm{T}}\end{array}$ & 71 & 36 & 46 & 25 & 43 & 100 & 6 & 4 \\
\hline 6. H. tsunoensis JCM $9105^{\mathrm{T}}$ & 70 & 9 & 13 & 7 & 8 & 7 & 100 & 5 \\
\hline $\begin{array}{l}\text { 7. Catelliglobosispora koreensis } \\
\text { JCM } 10976^{\mathrm{T}}\end{array}$ & 70 & 8 & 11 & 5 & 6 & 6 & 2 & 100 \\
\hline
\end{tabular}


$\mathrm{C}_{18: 1} \omega 8 c$ and $\mathrm{C}_{15: 0}$ were present in the fatty acid profile of Catellatospora tsunoense (Table 1). The mean DNA G + C contents of Catellatospora koreensis and Catellatospora tsunoense were 70.4 and $70.3 \mathrm{~mol} \%$, respectively (Table 5).

The phylogenetic analysis, DNA-DNA hybridization studies, and chemical and morphological characteristics show that Catellatospora koreensis LM $042^{\mathrm{T}}$ and Catellatospora tsunoense $6420-\mathrm{P}^{\mathrm{T}}$ can be differentiated from each other and from other members of the genus Catellatospora and other genera of the family Micromonosporaceae. Therefore, it is proposed that these organisms be classified as representing two new genera. On the basis of phenotypic and genotypic data, Catelliglobosispora koreensis gen. nov., comb. nov. and Hamadaea tsunoensis gen. nov., comb. nov. are proposed.

\section{Description of Catelliglobosispora gen. nov.}

Catelliglobosispora [Ca.tel.li.glo'bo.si.spo'ra. L. n. catella small chain; L. adj. globosus spherical; Gr. fem. n. spora a seed and in biology a spore; N.L. fem. n. Catelliglobosispora (organism forming) small chain and spherical spores].

The description is based on data taken from previous studies (Lee et al., 2000; Ara \& Kudo, 2006) and this study. Aerobic. Gram-positive. Catalase-positive. Urease-negative. Nitrate is reduced to nitrite. $\mathrm{H}_{2} \mathrm{~S}$ is not produced. Mesophilic. Vegetative hyphae are branched but not fragmented. Aerial mycelia are absent. Short chains of spores are borne directly on substrate mycelium. Spores are cylindrical and non-motile and spore surfaces are smooth. Substrate mycelia are cream to light yellow. Globose bodies are formed. Soluble pigments are not produced. Grow at $\mathrm{pH}$ 6.0-9.0. Temperature range for growth is $20-37^{\circ} \mathrm{C}$. Cell walls contain meso-DAP, rhamnose, ribose, galactose, xylose, mannose and glucose (type II cell wall and a variant of sugar pattern D). Acyl type of muramic acid is glycolyl. Predominant menaquinone is $\mathrm{MK}-10\left(\mathrm{H}_{4}\right)$, with minor amounts of MK-9 $\left(\mathrm{H}_{4}\right)$ and MK-10 $\left(\mathrm{H}_{6}\right)$. Diagnostic phospholipids comprise PE, DPG and PI. Glucosaminecontaining phospholipid and phosphatidylcholine are absent. Mycolic acids are absent. Predominant fatty acids $\left(>10.0 \%\right.$ of total) are iso- $\mathrm{C}_{16: 0}$ and iso- $\mathrm{C}_{14: 0}$; significant amounts of saturated, unsaturated and anteiso-branched fatty acids are also present. The $\mathrm{G}+\mathrm{C}$ content of the DNA of the type species is $70.4 \%$ (determined by HPLC). The type species is Catelliglobosispora koreensis.

\section{Description of Catelliglobosispora koreensis comb. nov.}

Catelliglobosispora koreensis (ko.re.en'sis. N.L. fem. adj. koreensis of Korea, pertaining to soil from Korea from which the organism was isolated).

Basonym: Catellatospora koreensis Lee et al. 2000.

Morphological, chemotaxonomic and general characteristics are given above for the genus description. The description is based on data taken from previous studies
(Lee et al., 2000) and our own studies. Straight, relatively short chains of non-motile spores are borne directly on substrate mycelium. Each spore is cylindrical and has a smooth surface. Abundant globose bodies are observed on oatmeal agar and ISP medium 4 . Good growth occurs on ISP medium 3, ISP medium 4, glucose-asparagine agar, glucose-yeast extract agar, Hickey-Tresner agar, yeast extract-starch agar and Bennett agar. Moderate growth occurs on yeast extract-malt extract agar, $1 / 5$ yeast extractstarch agar, oatmeal-nitrate agar and poor growth occurs on glycerol-asparagine agar, nutrient agar, ISP medium 7, tap-water agar and sucrose-nitrate agar. Melanin pigment production on ISP medium 7 is negative. Abundant sporulation occurs on tap-water agar and sucrose-nitrate agar and moderate sporulation occurs on ISP medium 7. In general, vegetative mycelia are cream to honey-gold in colour and aerial mycelia are not present. Utilizes Larabinose, cellobiose, D-glucose, D-galactose, D-mannose, maltose, D-fructose, D-xylose, D-lactose, sucrose, starch and raffinose as carbon sources. Does not utilize D-arabinose, dextran, gluconate, inulin, melezitose, melibiose, glycerol, erythritol, D-ribose, L-rhamnose, salicin, L-sorbose, myoinositol, meso-inositol, 2-propanol, propanol, D-mannitol, sorbitol, D-xylitol, adonitol, dulcitol, butanol, meso-erythritol, ethanol, methyl $\alpha$-D-glucoside or trehalose. Does not degrade adenine, hippurate, hypoxanthine, DL-tyrosine or xanthine. Hydrolyses casein, gelatin and starch, but not DNA. Grows on $0.001 \%$ brilliant green and $0.0001 \%$ crystal violet. No growth in $3 \% \mathrm{NaCl}$ and $0.01 \%$ lysozyme. Susceptible to novobiocin $\left(50 \mu \mathrm{g} \mathrm{ml} \mathrm{m}^{-1}\right)$, vancomycin $\left(20 \mu \mathrm{g} \mathrm{ml}^{-1}\right)$, streptomycin $\left(100 \mu \mathrm{g} \mathrm{ml}^{-1}\right)$ and tetracycline $\left(10 \mu \mathrm{g} \mathrm{ml}^{-1}\right)$, and resistant to gentamicin $\left(50 \mu \mathrm{g} \mathrm{ml}^{-1}\right)$ and neomycin $\left(5 \mu \mathrm{g} \mathrm{ml}^{-1}\right)$. Predominant fatty acids are iso- $\mathrm{C}_{16: 0}(44.5 \%)$, iso- $\mathrm{C}_{14: 0}(10.8 \%)$, iso- $\mathrm{C}_{16: 1}(8.6 \%)$, iso- $\mathrm{C}_{15: 0}(8.5 \%)$, with minor amounts of anteiso- $\mathrm{C}_{17: 0}$ $(6.6 \%)$, anteiso- $\mathrm{C}_{15: 0}(3.7 \%)$, iso- $\mathrm{C}_{18: 0}(3.0 \%), 2-\mathrm{OH}-$ $\mathrm{C}_{16: 1}(2.9 \%), \mathrm{C}_{18: 0}(2.6 \%)$, iso- $\mathrm{C}_{17: 0}(1.7 \%)$, anteiso$\mathrm{C}_{17: 1} \omega 9 c(1.5 \%), \mathrm{C}_{16: 0}(1.2 \%)$ and $\mathrm{C}_{17: 0}(1.2 \%)$.

The type strain, LM $042^{\mathrm{T}} \quad\left(=\mathrm{JCM} \quad 10976^{\mathrm{T}}=\mathrm{DSM}\right.$ $44566^{\mathrm{T}}=$ IMSNU $50729^{\mathrm{T}}$ ), was isolated from soil from a gold-mine cave.

\section{Description of Hamadaea gen. nov.}

Hamadaea (Ha.ma.da.e'a. N.L. fem. n. Hamadaea of Hamada, named after Masa Hamada, the Japanese microbiologist who made a tremendous contribution to actinomycete research).

The description is based on data from previous studies (Asano et al., 1989; Lee et al., 2000) and our own studies. Aerobic. Gram-positive. Catalase-positive. Urease-negative. Nitrate is reduced to nitrite. $\mathrm{H}_{2} \mathrm{~S}$ is not produced. Mesophilic. Vegetative hyphae are branched but not fragmented. Aerial mycelia are absent. Short chains of spores are borne directly on substrate mycelium. 3-O-Methyl-rhamnose is lacking in the cell walls. Require thiamine for growth. Cell walls contain meso- and 3$\mathrm{OH}-\mathrm{DAP}$, xylose, galactose, mannose, ribose, arabinose, 
rhamnose and glucose (type II cell wall and a variant of sugar pattern D). Acyl type of muramic acid is glycolyl. Major menaquinone is MK-9 $\left(\mathrm{H}_{6}\right)$, with minor amounts of MK9( $\left.\mathrm{H}_{4}\right)$ and MK-9 $\left(\mathrm{H}_{8}\right)$. Diagnostic phospholipids include phosphatidylethanolamine, OH-PE, DPG, PI and PIMs. Glucosamine-containing phospholipid and phosphatidylcholine are absent. Mycolic acids are absent. The $\mathrm{G}+\mathrm{C}$ content of the DNA of the type species is $70.3 \%$ (as determined by HPLC). Member of the family Micromonosporaceae. The type species is Hamadaea tsunoensis.

\section{Description of Hamadaea tsunoensis comb. nov.}

Hamadaea tsunoensis (tsu.no.en'sis. N.L. fem. adj. tsunoensis of Tsuno-gun, Yamaguchi, Japan, the origin of the soil sample from which the type strain was isolated).

\section{Basonym: Catellatospora tsunoense Asano et al. 1989.}

Morphological, chemotaxonomic and general characteristics are given above for the genus description. The description is based on data taken from previous studies (Asano et al., 1989 ) and our own studies. Good growth occurs on ISP medium 2, ISP medium 3, Bennett agar, 1/5 yeast-starch agar, glucose-yeast extract agar and yeast extract-starch agar. Moderate growth occurs on oatmeal-nitrate agar, HickeyTresner agar and poor growth on glucose-asparagine agar, glycerol-asparagine agar, ISP medium 4, ISP medium 7 , nutrient agar, tap-water agar and sucrose-nitrate agar. Colony mass is pale yellow to bright marigold. Spores are cylindrical and non-motile. Spore surface is smooth. Abundant globose bodies are present on tap-water agar medium and are $0.4-0.6 \mu \mathrm{m}$ in diameter. Abundant sporulation occurs on tap-water agar and moderate sporulation on glycerol-asparagine and 1/5 yeast extractstarch agar medium. Sporulates on tap-water agar medium in 10 days to 1 month at $28{ }^{\circ} \mathrm{C}$. No soluble and melanin-like pigments are produced. No growth occurs in the presence of $3 \% \mathrm{NaCl}$, lysozyme $(0.005 \%)$, crystal violet $(0.001 \%)$ or sodium lauryl sulfate $(0.01 \%)$. Susceptible to 6-demethyltetracycline $\left(500 \mu \mathrm{g} \mathrm{ml}^{-1}\right)$, novobiocin $\left(50 \mu \mathrm{g} \mathrm{ml}^{-1}\right)$, gentamicin $\left(50 \mu \mathrm{g} \mathrm{ml} l^{-1}\right)$, streptomycin $\left(100 \mu \mathrm{g} \mathrm{ml}{ }^{-1}\right)$ and vancomycin $\left(50 \mu \mathrm{g} \mathrm{ml}^{-1}\right)$, and resistant to neomycin $\left(5 \mu \mathrm{g} \mathrm{ml}^{-1}\right)$. Does not liquefy gelatin or peptonize milk. Positive for activities of leucine arylamidase, $\beta$-galactosidase, $\alpha$-glucosidase and $\beta$-glucosidase, but low or negative for activities of cystine arylamidase, $\alpha$-fucosidase and $\beta$ glucuronidase. Utilizes L-arabinose, D-xylose, D-fructose, Lrhamnose, $\alpha$-melibiose, sucrose, raffinose, trehalose, cellobiose, D-galactose, D-glucose, maltose, lactose, mannose and starch. Poor growth occurs on glycerol, salicin and no growth on erythritol, adonitol, myo-inositol, dextran, methyl $\alpha$-D-glucoside, D-ribose or D-mannitol. Predominant fatty acids are iso- $\mathrm{C}_{15: 0}(29.5 \%)$, anteiso$\mathrm{C}_{17: 0}(15.6 \%)$, iso- $\mathrm{C}_{16: 0}(10.4 \%)$, anteiso- $\mathrm{C}_{15: 0}(8.8 \%)$ and $\mathrm{C}_{17: 0}(7.6 \%)$, with small amounts of iso- $\mathrm{C}_{17: 0}(5.4 \%)$, 10-methyl-C $\mathrm{C}_{17: 0}$ (3.6\%), $\mathrm{C}_{16: 0} \quad(3.1 \%), \mathrm{C}_{18: 0} \quad(2.9 \%)$, $\mathrm{C}_{17: 1} \omega 6 c(2.7 \%)$, summed feature $3(2.0 \%), \mathrm{C}_{17: 1} \omega 8 c$ (1.7\%), 10-methyl- $\mathrm{C}_{18: 0}(1.5 \%)$ and $\mathrm{C}_{15: 0}(1.0 \%)$.
The type strain, $\quad 6420-\mathrm{P}^{\mathrm{T}} \quad\left(=\mathrm{JCM} \quad 9105^{\mathrm{T}}=\mathrm{DSM}\right.$ $44101^{\mathrm{T}}=$ IMSNU $22005^{\mathrm{T}}$ ), was isolated from woodland soil.

\section{Emended description of Catellatospora Asano and Kawamoto 1986 emend. Lee and Hah 2002}

Aerobic. Gram-positive. Catalase-positive. Urease-negative. Nitrate is reduced to nitrite. $\mathrm{H}_{2} \mathrm{~S}$ is not produced. Mesophilic. Vegetative hyphae are branched but not fragmented. Colony masses are bright yellow. Aerial mycelia are not formed. Chains of non-motile spores arise singly or in tufts from vegetative hyphae on the surface of agar media. Globose bodies are not produced. Contain meso- and 3-OH-DAP as diamino acids, a glycolylated peptidoglycan and whole-cell sugars xylose, mannose, galactose, arabinose, ribose and glucose. Rhamnose is variable according to species. Predominant menaquinone is MK-9 $\left(\mathrm{H}_{4}\right)$. PE, DPG, PI and PIMs are present as diagnostic phospholipids. Fatty acid pattern is type 3 b. Mycolic acids are absent. The G+C content of the DNA is 70.71$71.44 \mathrm{~mol} \%$. Members of this genus form a coherent group within the radiation of the family Micromonosporaceae on the basis of $16 \mathrm{~S}$ rRNA sequence data. The type species is Catellatospora citrea.

\section{Acknowledgements}

I. A. was supported financially by the Japan Society for the Promotion of Science (JSPS) for her research fellowship. We are very grateful to Dr J. P. Euzéby for the nomenclature of the two genera.

\section{References}

Ara, I. \& Kudo, T. (2006). Three novel species of the genus Catellatospora, Catellatospora chokoriensis sp. nov., Catellatospora coxensis sp. nov. and Catellatospora bangladeshensis sp. nov. and transfer of Catellatospora citrea subsp. methionotrophica Asano and Kawamoto 1988 to Catellatospora methionotrophica sp. nov., comb. nov. Int J Syst Evol Microbiol 56, 393-400.

Ara, I. \& Kudo, T. (2007a). Luedemannella gen. nov., a new genus of the family Micromonosporaceae and description of Luedemannella helvata sp. nov. and Luedemannella flava sp. nov. J Gen Appl Microbiol 53, 39-51.

Ara, I. \& Kudo, T. (2007b). Two new species of the genus Micromonospora: Micromonospora chokoriensis sp. nov. and Micromonospora coxensis sp. nov., isolated from sandy soil. J Gen Appl Microbiol 53, 29-37.

Ara, I. \& Kudo, T. (2007c). Sphaerisporangium gen. nov., a new genus of the family Streptosporangiaceae with descriptions of three new species as Sphaerisporangium melleum sp. nov., Sphaerisporangium rubeum sp. nov., Sphaerisporangium cinnabarinum sp. nov. and transfer of Streptosporangium viridialbum Nonomura and Ohara 1960 to Sphaerisporangium viridialbum comb. nov. Actinomycetologica 21, 11-21.

Ara, I. \& Kudo, T. (2007d). Krasilnikovia gen. nov., a new genus of the family Micromonosporaceae and description of Krasilnikovia cinnamomea sp. nov. Actinomycetologica 21, 1-10.

Ara, I., Kudo, T., Matsumoto, A., Takahashi, Y. \& Omura, S. (2007a). Nonomuraea bangladeshensis sp. nov., and Nonomuraea coxensis sp. nov. Int J Syst Evol Microbiol 57, 1504-1509. 
Ara, I., Kudo, T., Matsumoto, A., Takahashi, Y. \& Omura, S. (2007b). Nonomuraea maheshkhaliensis sp. nov., a novel actinomycete isolated from mangrove rhizosphere mud. J Gen Appl Microbiol 53, 159-166.

Asano, K. \& Kawamoto, I. (1986). Catellatospora, a new genus of the Actinomycetales. Int J Syst Bacteriol 36, 512-517.

Asano, K. \& Kawamoto, I. (1988). Catellatospora citrea subsp. methionotrophica subsp. nov., a methionine-deficient auxotroph of the Actinomycetales. Int J Syst Bacteriol 38, 326-327.

Asano, K., Masunaga, I. \& Kawamoto, I. (1989). Catellatospora matsumotoense sp. nov. and C. tsunoense sp. nov., actinomycetes found in woodland soils. Int J Syst Bacteriol 39, 309-313.

Collins, M. D., Pirouz, T., Goodfellow, M. \& Minnikin, D. E. (1977). Distribution of menaquinones in actinomycetes and corynebacteria. J Gen Microbiol 100, 221-230.

Collins, M. D., Faulkner, M. \& Keddie, R. M. (1984). Menaquinone composition of some spore forming actinomycetes. Syst Appl Microbiol 5, 20-29.

Couch, J. N. (1950). Actinoplanes a new genus of the Actinomycetales. J Elisha Mitchell Sci Soc 66, 87-92.

Ezaki, T., Hashimoto, Y. \& Yabuuchi, E. (1989). Fluorometric deoxyribonucleic acid- deoxyribonucleic acid hybridization in microdilution wells as an alternative to membrane filter hybridization in which radioisotopes are used to determine genetic relatedness among bacterial strains. Int J Syst Bacteriol 39, 224-229.

Felsenstein, J. (1985). Confidence limits on phylogenies: an approach using the bootstrap. Evolution 39, 783-791.

Goodfellow, M. L., Stanton, J., Simpson, K. E. \& Minnikin, D. E. (1990). Numerical and chemical classification of Actinoplanes and some related actinomycetes. J Gen Microbiol 136, 19-36.

Horan, A. C. \& Brodsky, B. (1986). Actinoplanes caeruleus sp. nov., a blue-pigmented species of the genus Actinoplanes. Int J Syst Bacteriol 36, 187-191.

Itoh, T., Kudo, T., Parenti, F. \& Seino, A. (1989). Amended description of the genus Kineosporia, based on chemotaxonomic and morphological studies. Int J Syst Bacteriol 39, 168-173.

Jacobson, E., Grauville, W. C. \& Fogs, C. E. (1958). Color Harmony Manual, 4th edn. Chicago: Container Corporation of America.

Kane, W. D. (1966). A new genus of Actinoplanaceae, Pilimelia, with a description of two species, Pilimelia terevasa and Pilimelia anulata. J Elisha Mitchell Sci Soc 82, 220-230.

Koch, C., Kroppenstedt, R. M., Rainey, F. A. \& Stackebrandt, E. (1996). 16S ribosomal DNA analysis of the genera Micromonospora, Actinoplanes, Catellatospora, Catenuloplanes, Couchioplanes, Dactylosporangium, and Pilimelia and emendation of the family Micromonosporaceae. Int J Syst Bacteriol 46, 765-768.

Kroppenstedt, R. M. (1985). Fatty acid and menaquinone analysis of actinomycetes and related organisms. In Chemical Methods in Bacterial Systematics, pp. 173-199. Edited by M. Goodfellow \& D. E. Minnikin. London: Academic Press.

Kudo, T., Matsushima, K., Itoh, T., Sasaki, J. \& Suzuki, K. (1998). Description of four new species of the genus Kineosporia: Kineosporia succinea sp. nov., Kineosporia rhizophila sp. nov., Kineosporia mikuniensis sp. nov. and Kineosporia rhamnosa sp. nov., isolated from plant samples, and amended description of the genus Kineosporia. Int J Syst Bacteriol 48, 1245-1255.

Kudo, T., Nakajima, Y. \& Suzuki, K. (1999). Catenuloplanes crispus (Petrolini et al. 1993) comb. nov.: incorporation of the genus Planopolyspora Petrolini 1993 into the genus Catenuloplanes Yokota et al. 1993 with an amended description of the genus Catenuloplanes. Int J Syst Bacteriol 49, 1853-1860.
Lechevalier, M. P., Stern, A. E. \& Lechevalier, H. A. (1981). Phospholipids in the taxonomy of actinomycetes. Zentbl Bakteriol Hyg Abt 1 (Suppl 11), 111-116.

Lee, S. D. \& Hah, Y. C. (2002). Proposal to transfer Catellatospora ferruginea and 'Catellatospora ishikariense' to Asanoa gen. nov. as Asanoa ferruginea comb. nov. and Asanoa ishikariensis sp. nov., with emended description of the genus Catellatospora. Int J Syst Evol Microbiol 52, 967-972.

Lee, S. D., Goodfellow, M. \& Hah, Y. C. (1999). A phylogenetic analysis of the genus Catellatospora based on 16S ribosomal DNA sequences, including transfer of Catellatospora matsumotoense to the genus Micromonospora as Micromonospora matsumotoense comb. nov. FEMS Microbiol Lett 178, 349-354.

Lee, S. D., Kang, S.-O. \& Hah, Y. C. (2000). Catellatospora koreensis sp. nov., a novel actinomycete isolated from a gold-mine cave. Int J Syst Evol Microbiol 50, 1103-1111.

Maldonado, L. A., Fenical, W., Jensen, P. R., Kauffman, C. A., Mincer, T. J., Ward, A. C., Bull, A. T. \& Goodfellow, M. (2005). Salinispora arenicola gen. nov., sp. nov. and Salinispora tropica sp. nov., obligate marine actinomycetes belonging to the family Micromonosporaceae. Int J Syst Evol Microbiol 55, 1759-1766.

Matsumoto, A., Takahashi, Y., Shinose, M., Seino, A., Iwai, Y. \& Omura, S. (2003). Longispora albida gen. nov., a novel genus of the family Micromonosporaceae. Int J Syst Evol Microbiol 53, 1553-1559.

Mikami, H. \& Ishida, Y. (1983). Post-column fluorometric detection of reducing sugar in high-performance liquid chromatography using arginine. Bunseki Kagaku 32, E207-E210.

Minnikin, D. E., O'Donnell, A. G., Goodfellow, M., Alderson, G., Athalye, M., Schaal, A. \& Parlett, J. H. (1984). An integrated procedure for the extraction of bacterial isoprenoid quinones and polar lipids. J Microbiol Methods 2, 233-241.

Nakajima, Y., Kitpreechavanich, V., Suzuki, K. \& Kudo, T. (1999). Microbispora corallina sp. nov., a new species of the genus Microbispora isolated from Thai soil. Int J Syst Bacteriol 49, 1761-1767.

Ørskov, J. (1923). Investigations into the Morphology of the Ray Fungi. Copenhagen: Levin and Munksgaard.

Raeder, U. \& Broda, P. (1985). Rapid preparation of DNA from filamentous fungi. Lett Appl Microbiol 1, 17-20.

Rheims, H., Schumann, P., Rohde, M. \& Stackebrandt, E. (1998). Verrucosispora gifhornensis gen. nov., sp. nov., a new member of the actinobacterial family Micromonosporaceae. Int J Syst Bacteriol 48, 1119-1127.

Saito, H. \& Miura, K. (1963). Preparation of transforming deoxyribonucleic acid by phenol treatment. Biochim Biophys Acta 72, 619-629.

Saitou, N. \& Nei, M. (1987). The neighbor-joining method: a new method for reconstructing phylogenetic trees. Mol Biol Evol 4, 406425.

Sasser, M. (1990). Identification of bacteria by gas chromatography of cellular fatty acids, MIDI Technical Note 101. Newark, DE: MIDI Inc.

Sharples, G. P. \& Williams, S. T. (1974). Fine structure of the globose bodies of Dactylosporangium thailandense (Actinomycetales). J Gen Microbiol 84, 219-222.

Shirling, E. B. \& Gottlieb, D. (1966). Methods for characterization of Streptomyces species. Int J Syst Bacteriol 16, 313-340.

Stackebrandt, E. \& Kroppenstedt, R. M. (1987). Union of the genera Actinoplanes Couch, Ampullariella Couch, and Amorphosporangium Couch in a redefined genus Actinoplanes. Syst Appl Microbiol 9, 110-114.

Staneck, J. L. \& Roberts, G. D. (1974). Simplified approach to identification of aerobic actinomycetes by thin-layer chromatography. Appl Microbiol 28, 226-231. 
Stevenson, I. L. (1967). Utilization of aromatic hydrocarbons by Arthrobacter spp. Can J Microbiol 13, 205-211.

Swofford, D. L. (2001). PAUP*: Phylogenetic analysis using parsimony ( ${ }^{*}$ and other methods), version 4. Sunderland, MA: Sinauer Associates.

Tamaoka, J. (1994). Determination of DNA base composition. In Chemical Methods in Prokaryotic Systematics, pp. 463-470. Edited by M. Goodfellow \& A. G. O’Donnell. Chichester: John Wiley \& Sons.

Tamaoka, J. \& Komagata, K. (1984). Determination of DNA base composition by reversed-phase high-performance liquid chromatography. FEMS Microbiol Lett 25, 125-128.

Tamaoka, J., Katayama-Fujimura, Y. \& Kuraishi, H. (1983). Analysis of bacterial menaquinone mixtures by high performance liquid chromatography. J Appl Bacteriol 54, 31-36.

Tamura, T. \& Hatano, K. (2001). Phylogenetic analysis of the genus Actinoplanes and transfer of Actinoplanes minutisporangius Ruan et al. 1986 and 'Actinoplanes aurantiacus' to Cryptosporangium minutisporangius comb. nov. and Cryptosporangium aurantiacum sp. nov. Int J Syst Evol Microbiol 51, 2119-2125.

Tamura, T., Nakagaito, Y., Nishii, T., Hasegawa, T., Stackebrandt, E. \& Yokota, A. (1994). A new genus of the order Actinomycetales, Couchioplanes gen. nov., with descriptions of Couchioplanes caeruleus (Horan and Brodsky 1986) comb. nov. and Couchioplanes caeruleus subsp. azureus subsp. nov. Int J Syst Bacteriol 44, 193-203.

Tamura, T., Hayakawa, M. \& Hatano, K. (1997). A new genus of the order Actinomycetales, Spirilliplanes gen. nov., with description of Spirilliplanes yamanashiensis sp. nov. Int J Syst Bacteriol 47, 97-102.

Tamura, T., Hayakawa, M. \& Hatano, K. (2001). A new genus of the order Actinomycetales, Virgosporangium gen. nov., with descriptions of Virgosporangium ochraceum sp. nov. and Virgosporangium aurantiacum sp. nov. Int J Syst Evol Microbiol 51, 1809-1816.
Tamura, T., Hatano, K. \& Suzuki, K. (2006). A new genus of the family Micromonosporaceae, Polymorphospora gen. nov., with description of Polymorphospora rubra sp. nov. Int J Syst Evol Microbiol 56, 1959 1964.

Thawai, C., Tanasupawat, S., Itoh, T. \& Kudo, T. (2006). Actinocatenispora thailandica gen. nov., sp. nov., a new member of the family Micromonosporaceae. Int J Syst Evol Microbiol 56, 1789-1794.

Thiemann, J. E., Pagani, H. \& Beretta, G. (1967). A new genus of the Actinoplanaceae: Dactylosporangium, gen. nov. Arch Mikrobiol 58, 4252.

Thompson, J. D., Gibson, T. J., Plewniak, F., Jeanmougin, F. \& Higgins, D. G. (1997). The CLUSTAL_X windows interface: flexible strategies for multiple sequence alignment aided by quality analysis tools. Nucleic Acids Res 25, 4876-4882.

Tomiyasu, I. (1982). Mycolic acid composition and thermally adaptative changes in Nocardia asteroides. J Bacteriol 151, 828-837.

Uchida, K. \& Aida, K. (1984). An improved method for the glycolate test for simple identification of acyl type of bacterial cell walls. J Gen Appl Microbiol 30, 131-134.

Vobis, G. (1989). Actinoplanetes. In Bergey's Manual of Systematic Bacteriology, vol. 4, pp. 2418-2450. Edited by S. T. Williams, M. E. Sharpe \& J. G. Holt. Baltimore: Williams \& Wilkins.

Waksman, S. A. (1950). The Actinomycetes: Their Nature, Occurrence, Activities and Importance. Waltham, MA: Chronica Botanica.

Waksman, S. A. (1961). Classification, identification and description of genera and species. In The Actinomycetes, vol. 2. Baltimore: Williams \& Wilkins.

Yokota, A., Tamura, T., Hasegawa, T. \& Huang, L. H. (1993). Catenuloplanes japonicus gen. nov., sp. nov., nom. rev., a new genus of the order Actinomycetales. Int J Syst Bacteriol 43, 805-812. 